\title{
Dynamic Stability Loss of Closed Circled Cylindrical Shells Estimation Using Wavelets
}

\author{
V.A. Krysko ${ }^{1}$, J. Awrejcewicz ${ }^{2}$, M. Zhigalov ${ }^{1}$, \\ V. Soldatov ${ }^{1}$, E.S. Kuznetsova ${ }^{1}$, S. Mitskevich ${ }^{1}$
}

${ }^{1}$ Saratov State Technical University, Department of Mathematics and Modeling, 410054, Polytechnical 77, Saratov, Russia, Email: $\underline{\text { tak@san.ru }}$

${ }^{2}$ Technical University of Lodz, Department of Automatics and Biomechanics, 1/15 Stefanowski St., 90-924 Lodz, Poland Email: awrejcew@p.lodz.pl

Abstract: In this work dynamic stability loss of closed cylindrical shells is studied. The hybrid type PDEs governing cylindrical shells dynamics and regarding deflection (Airy's) function and stresses are first derived. Then they are reduced to ODEs and algebraic equations (AE) applying the Bubnov-Galerkin high order approximations method. Dynamic stability loss of the cylindrical shells subject to sign-changeable loading using wavelets is investigated. In addition, the convergence of the Bubnov-Galerkin method and results validation are addressed.

Keywords: chaos, closed cylindrical shells, wavelets, nonlinear dynamics, dynamic stability loss.

\section{Introduction}

Closed cylindrical shells are members of various constructions and machines including airplanes, ships, civil engineering constructions, measurement devices, etc. For many years thousands of researchers attacked a key problem regarding safe and durable properties of thin- and thick-walled constructions addressing and formulating the so called stability loss criteria (see [1]-[4]). Note that even a particular case of this topic, i.e. analysis of free and forced nonlinear vibrations of circled cylindrical shells attracted and still attracts many engineering oriented researchers (see for instance [5-10] and the cited references therein).

In this work, among others, we propose a novel stability loss criterion with a help of the wavelet-based analysis.

\section{Problem statement}

We study the closed circled cylindrical shell of finite length with constant stiffness and density subject to action of non-uniform sign-changeable load and being embedded in a temperature field (Fig. 1). The following coordinates are introduced: axis $y$ refers to circled co-ordinate, whereas axis $z$ 
Krysko, V. A., Awrejcewicz J. et al.

overlaps a normal one of the shell's middle surface. Therefore, our shell is defined as $\Omega=\{x, y, z \mid(x, y) \in[0 ; L] \times[0 ; 2 \pi],-h \leq z \leq h\}$.

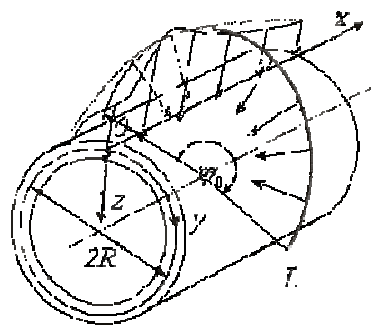

Fig. 1. Computational scheme

Its governing dynamic non-dimensional equations have the following form $[9,10]$ :

$$
\begin{aligned}
& \frac{1}{12\left(1-v^{2}\right)}\left[\lambda^{-2} \frac{\partial^{4} w}{\partial x^{4}}+2 \frac{\partial^{4} w}{\partial x^{2} \partial y^{2}}+\lambda^{2} \frac{\partial^{4} w}{\partial y^{4}}\right]-L(w, F) \\
& -k_{y} \frac{\partial^{2} F}{\partial x^{2}}-\frac{\partial^{2} w}{\partial t^{2}}-\varepsilon \frac{\partial w}{\partial t}+k_{y}^{2} q(x, y, t)=0, \\
& \left\{\lambda^{-2} \frac{\partial^{4} F}{\partial x^{4}}+2 \frac{\partial^{4} F}{\partial x^{2} \partial y^{2}}+\lambda^{2} \frac{\partial^{4} F}{\partial y^{4}}+\frac{1}{2} L(w, w)+k \frac{\partial^{2} w}{\partial x^{2}}\right\}=0 .
\end{aligned}
$$

Equations (1) have been transformed to the non-dimensional form using the following relations:

$$
w=2 h \bar{w}, F=E_{0}(2 h)^{3} \bar{F}, t=\frac{R L}{2 h \sqrt{g E_{0}}} t, \lambda=L / R ; x=L \bar{x}, y=R \bar{y} ;
$$
$k_{y}=\bar{k}_{y} \frac{2 h}{R^{2}}, q=\bar{q} \frac{E_{0}(2 h)^{4}}{L^{2} R^{2}}$. Here $L$ and $R=R_{y}$ are shell length and radius, respectively; $t$ - time, $\varepsilon$ - damping coefficient of a shell surrounding medium, $F$ - stress (Airy's) function, $w$ - shell's deflection, $h$ - shell's thickness, $v$ - Poisson's coefficient, $g$ - gravity acceleration, $E_{0}$ - shell material Young modulus, $k_{y}$-shell's curvature regarding axis $y$ and finally 


$$
\begin{aligned}
& L(w, F)=\frac{\partial^{2} w}{\partial x^{2}} \frac{\partial^{2} F}{\partial y^{2}}+\frac{\partial^{2} w}{\partial y^{2}} \frac{\partial^{2} F}{\partial x^{2}}-2 \frac{\partial^{2} w}{\partial x \partial y} \frac{\partial^{2} F}{\partial x \partial y}, \\
& L(w, w)=2\left[\frac{\partial^{2} w}{\partial x^{2}} \frac{\partial^{2} w}{\partial y^{2}}-\left(\frac{\partial^{2} w}{\partial x \partial y}\right)^{2}\right]
\end{aligned}
$$

are widely known nonlinear operators (note that bars over non-dimensional quantities are omitted).

Let us focus on investigation of one of the boundary conditions, i.e. hinged shell's support with flexible ribs on the shell's edges:

$$
w=M_{x}=N_{x}=\varepsilon_{y}=0 \text { for } x=0 ; 1, y=0 ; 2 \pi,
$$

and with the following initial conditions:

$$
\left.w(x, y)\right|_{t=0}=0,\left.\quad \frac{\partial w}{\partial t}\right|_{t=0}=0 .
$$

The boundary value problem formulated so far is solved via the Bubnov-Galerkin method with high order approximations. In this work the being sought functions $w$ and $F$ are approximated by analytical expressions having a finite number of parameters arbitrarily taken through the following functions depending on time and spatial co-ordinates:

$$
w=\sum_{i=0}^{N_{1}} \sum_{j=0}^{N_{2}} A_{i j}(t) \phi_{i j}(x, y), \quad F=\sum_{i=0}^{N_{1}} \sum_{j=0}^{N_{2}} B_{i j}(t) \psi_{i j}(x, y),
$$

where $\phi_{i j}(x, y)$ and $\psi_{i j}(x, y)$ are certain functions of $\mathrm{x}, \mathrm{y}$.

After the Bubnov-Galerkin method application to system (1), a system of ODEs is obtained regarding functions $A_{i j}(t)$ and $B_{i j}(t)$, and its matrix form is:

$$
\begin{gathered}
\mathbf{G}(\ddot{A}+\varepsilon \dot{A})+\mathbf{S} A+\mathbf{C}_{1} B+\mathbf{D}_{1} A B=\mathbf{Q}_{q}(t), \\
\mathbf{C}_{2} A+\mathbf{P} B+\mathbf{D}_{2} A A=0,
\end{gathered}
$$

where $\quad \mathbf{G}=\left\|G_{i j k l}\right\|, \quad \mathbf{S}=\left\|S_{i j r s k l}\right\|, \quad \mathbf{C}_{1}=\left\|C_{1 i j k l}\right\|, \quad \mathbf{C}_{2}=\left\|C_{2 i j k l}\right\|$, $\mathbf{D}_{1}=\left\|D_{1 i j r s k l}\right\|, \mathbf{D}_{2}=\left\|D_{2 i j r s k l}\right\|, \mathbf{P}=\left\|P_{i j k l}\right\|-$ squared matrices of dimensions 
Krysko, V. A., Awrejcewicz J. et al.

$2 \cdot N_{1} \cdot N_{2} \times 2 \cdot N_{1} \cdot N_{2} ; \mathbf{A}=\left\|A_{i j}\right\|, \mathbf{B}=\left\|B_{i j}\right\|, \mathbf{Q}=\left\|Q_{i j}\right\|-$ matrices of dimensions $2 \cdot N_{1} \cdot N_{2} \times 1$.

Second equation of (5) is solved regarding matrix $\mathbf{B}$ using an inversed matrix method on each time step:

$$
\mathbf{B}=\left[-\mathbf{P}^{-1} \mathbf{D}_{2} \mathbf{A}-\mathbf{P}^{-1} \mathbf{C}_{2}\right] \mathbf{A} \text {. }
$$

Multiplying first equation of (5) by $\mathbf{G}^{-1}$ and denoting $\dot{A}=\mathbf{R}$, one achieves the Cauchy's problem for the following first order ODEs:

$$
\left\{\begin{array}{l}
\dot{\mathbf{R}}=-\bar{\varepsilon} \mathbf{R}+\mathbf{G}^{-1} \mathbf{D}_{1} A B-\mathbf{G}^{-1} \mathbf{S} A+q(\bar{t}) \mathbf{G}^{-1} \mathbf{Q} \\
\dot{A}=\mathbf{R}
\end{array}\right.
$$

These transformations are allowed since matrices $\mathbf{G}^{-1}$ and $\mathbf{P}^{-1}$ exist assuming linear independence of the co-ordinate functions.

We add to equations (7) boundary and initial conditions and the yielded Cauchy problem is solved using the fourth order Runge-Kutta algorithm (computational step is chosen via the Runge principle).

In order to satisfy boundary conditions (2), functions $\phi_{i j}, \psi_{i j}$ appeared in (4) are sought in the form of a product of two functions, where each of them depends only on one argument:

$$
\begin{aligned}
& w=\sum_{i=1}^{N_{1}} \sum_{j=0}^{N_{2}} A_{i j}(t) \sin (i \pi x) \cos (j y), \\
& F=\sum_{i=1}^{N_{1}} \sum_{j=0}^{N_{2}} B_{i j}(t) \sin (i \pi x) \cos (j y)
\end{aligned} .
$$

\section{Numerical results}

We study our shells' vibrations character using the following fixed parameters: $k_{y}=112.5$ for $\lambda=2, \varepsilon=9$, and subject to action of transversal load $q(t)=q_{0} \sin \left(\omega_{p} t\right)$ distributed on the whole shell's surface $(0 \leq x \leq 1$, $\omega_{p}=2.3$ ), and we take numbers of functions $N_{1}=1, N_{2}=9$ (note that increase of those numbers do not increase the results accuracy). 
Dynamic Stability Loss of Closed Circled Cylindrical Shells

Qualitative analysis is carried out using the well-known characteristics: $w(0.5 ; 0 ; t)$, wavelets in $2 \mathrm{D}$ and $3 \mathrm{D}$, phase portraits, Poincaré maps, FFT (Fast Fourier Transform), Lyapunov exponents and the autocorrelation functions. In particular, we focus on dynamic stability loss estimation of our investigated shell. Further, only the following characteristics will be reported: time histories, phase portraits, frequency power spectra, wavelet-spectra, and shell buckling forms for $0 \leq x \leq 1 ; 0 \leq y \leq 2 \pi$ and for $x=0.5 ; 0 \leq y \leq 2 \pi$.

In order to construct the dependence $w_{\max }\left(q_{0}\right)$, the computations have been carried out for $q_{0} \in[0 ; 0.4975]$. In addition, for each element $q_{0}$ a maximum shell deflection in point $(0,5 ; 0)$ has been estimated. Results are reported in Fig. 2, where one may observe the so-called shell dynamic stability loss (small change of $q_{0}$ is associated with a rapid shell deflection increase). Inflexion point of the curve given in Fig. 2 allows to define the critical value $q_{0}^{c r}$. We study the vibration character in the shell pre-critical state ( $q_{0} \leq 0.225$, see Figures 3-8). Analysis of shell vibrations (for instance, in point $\mathrm{A}$ ) allows to conclude that they are harmonic.

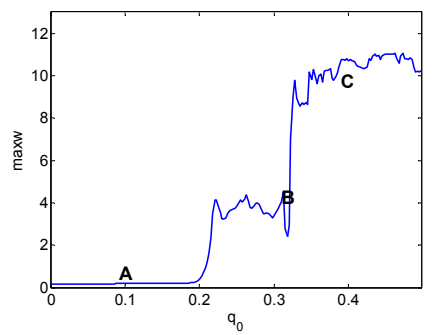
A: $q_{0}=0.1$ - pre-critical shell state;
B: $q_{0}=0.3225$ - after
first shell buckling;
C: $q_{0}=0.3975$ - after
second shell buckling.

Fig. 2. Relation $w_{\text {max }}\left(q_{0}\right)$

$$
q_{0}=0.1
$$


Krysko, V. A., Awrejcewicz J. et al.

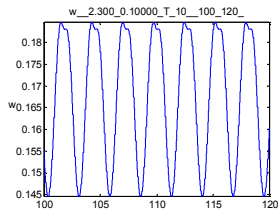

Fig. 3. $w(0.5 ; 0 ; t)$

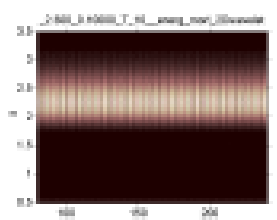

Fig. 4. 2D Morlet wavelet

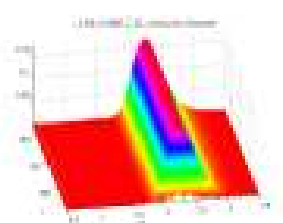

Fig. 5. 3D Morlet wavelet

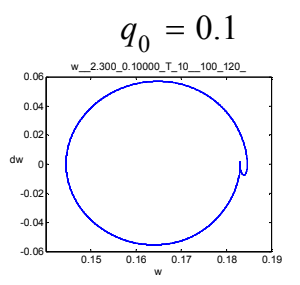

Fig. 6. Phase portrait $w(0.5 ; 0 ; t)$, $\dot{w}(0.5 ; 0 ; t)$

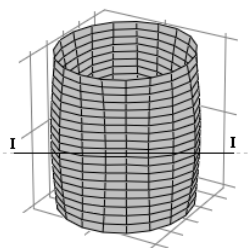

Fig. 7. Shell deflection form

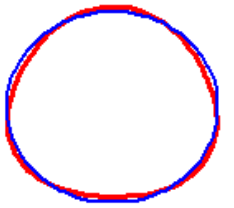

Fig. 8. Shell transversal cross-section I-I

Observe that when our dynamical system passed through the critical point $q_{0}^{c r}$ (we take $q_{0}=0.3225$ ), chaotic dynamics appears (see Figures 9-14).

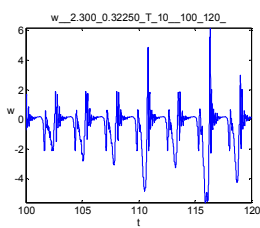

Fig. 9. Time history $w(0.5 ; 0 ; t)$

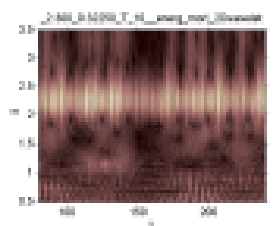

Fig. 10. 2D Morlet wavelet

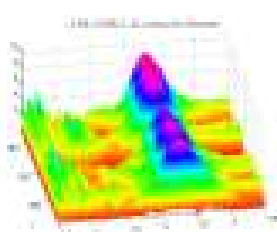

Fig. 11. 3D Morlet wavelet 


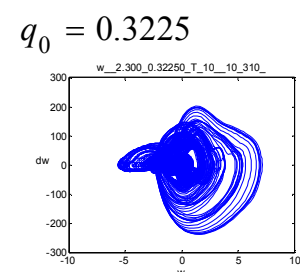

Fig. 12. Phase portrait $w(0.5 ; 0 ; t)$, $\dot{w}(0.5 ; 0 ; t)$

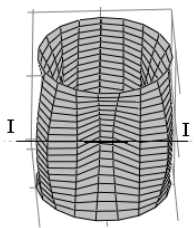

Fig. 13. Shell deflection form

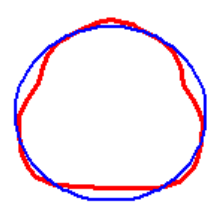

Fig. 14. Shell transversal cross-section I-I

In point $\mathrm{C}$ associated with second shell buckling $\left(q_{0}=0.3975\right)$ the wavelet-spectrum allows to detect an interesting qualitative change of the shell dynamics in a very short time interval $(t \approx 200)$.

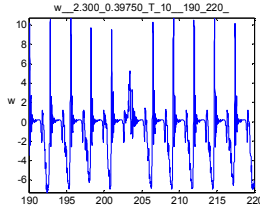

Figure 15. Time history $w(0.5 ; 0 ; t)$

$q_{0}=0.3975$

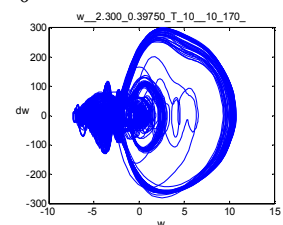

Figure 18. Phase portrait $w(0.5 ; 0 ; t)$, $\dot{w}(0.5 ; 0 ; t)$

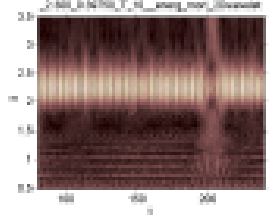

Figure 16. 2D Morlet wavelet

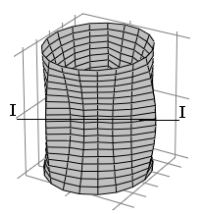

Figure 19. Shell deflection form

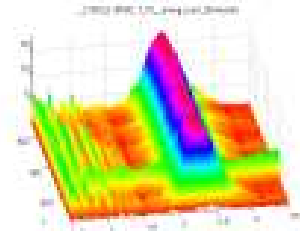

Figure 17. 3D Morlet wavelet

\section{Conclusions}

Owing to our computational technique used to study non-linear vibrations of 
Krysko, V. A., Awrejcewicz J. et al.

closed circled cylindrical shells subject to action of transversal signchangeable load the following conclusions are carried out: (i) our investigated shell exhibits dynamics stability loss during a transition from regular to chaotic vibrations; (ii) we have detected the second type shell buckling associated with chaotic intermittency behavior.

\section{References}

1. A. C. Shian, T. T. Soong and D. S. Roth. Dynamic buckling of conical shells with Imperfection. AIAA Journal, 12(6):24-30, 1974.

2. B. Budiansky and D. S. Roth. Axisymmetric Dynamic Buckling of Clamped Shallow Spherical Shells. NASA TN D-1510, 597-606, 1962.

3. B. Ya. Kantor. Nonlinear Problems of Theory of Non-Homogenous Shallow Shells. Naukowa Dumka, Kiev, 1971, in Russian.

4. V. A. Krysko and A. V. Krysko. Problems of bifurcations and stiff stability loss in nonlinear theory of plates. Mechanics of Plates and Shells in XXI century. The Saratov Technical University Press, 5067, 1999, in Russian.

5. H. N. Chu. Influence of large amplitude on flexural vibrations of thin cylindrical shell. J. Aerospace Sci., 28(8):602-609, 1961.

6. J. L. Novinski. Nonlinear transverse vibrations of orthotropic cylindrical shells. AIAA J., 1(3):617-620, 1963.

7. D. A. Evensen. Some observations on the nonlinear vibrations of thin cylindrical shells. AIAA J,. 1(12):2857-2858, 1963.

8. M. Amabili, F. Pellicano and M.P. Paidoussis. Non-linear vibration of simply supported circular cylindrical shells coupled to quiescent fluid. Journal of Fluids and Structures, 12:883-918, 1998.

9. V. A. Krysko, J. Awrejcewicz, E. S. Kusnetsova and A. V. Krysko. Chaotic vibrations of closed cylindrical shells in a temperature field. International Journal of Bifurcation and Chaos, 18(5):1515-1529, 2008.

10. V. A. Krysko, J. Awrejcewicz, E. S. Kusnetsova and A. V. Krysko Chaotic vibrations of closed cylindrical shells in a temperature field. Shock and Vibration, 15:335-343, 2008. 